Rev Chil Salud Pública 2012; Vol 16 (2): 115-122 Artículo original
CARMEN G. HERRERA(1), JOHANNA MAYR S. ${ }^{(2)}$, MARÍA PAZ BUSTOS ${ }^{(3)}$, ANDRÉS $\mathrm{CHILO}^{(3)}$, NIVIA JEFY(4) y KAREN LENGWENUS ${ }^{(5)}$

(1)Escuela de Nutrición y Dietética. Facultad de Ciencias de la Salud. Universidad Santo Tomás. Ejército 146 Santiago. Chile. cherrerag@ust.cl (2)Universidad Tecnológica. Osorno. Chile. (3)Médico General APS. Chile. ${ }^{(4)}$ Centro de Salud Familiar de Rahue Alto. Osorno. Chile. ${ }^{(5)}$ Nutricionista. Osorno. Chile.

Este estudio fue financiado por el Fondo Nacional de Investigación y Desarrollo en Salud (Fonis).

Proyecto SAO7120002.

\section{Estado nutricional y factores de riesgo cardiovascular en mujeres mapuche huilliche de la Provincia de Osorno}

\section{RESUMEN}

Objetivos: Para disponer de información desagregada de la situación nutricional y presencia de factores de riesgo cardiovascular de la mujer mapuche huilliche y conformar una línea diagnóstica que sirva de base para estudios posteriores e iniciativas que la incluyan, se estudió una muestra estadísticamente representativa de mujeres de esa etnia, jefas de hogar, de zonas rurales y urbanas de la Provincia de Osorno. Material y método: Se utilizó un diseño no experimental, transeccional y descriptivo. Resultados: Se encontró que un $48,8 \%$ presenta un $I M C>30$, superando en 18,1 puntos porcentuales el promedio nacional equivalente. Un $42,1 \%$ presenta obesidad abdominal (circunferencia cintura $>83 \mathrm{~cm}$ ) y el $73,5 \%$ tiene valores de alto riesgo en las razones de cintura-cadera y de cintura-estatura. Se presenta la relación de estos indicadores con variables como edad, ruralidad, presión arterial y reporte de enfermedades crónicas no transmisibles. Conclusiones: Se encontró alta prevalencia de factores de riesgo cardiovascular y baja concordancia entre IMC y percepción de estado nutricional.

Palabras clave: estado nutricional. mujer etnia mapuche huilliche, riesgo cardiovascular.

\section{ABSTRACT}

NUTRICIONAL STATE AND CARDIOVASCULAR RISK FACTORS IN MAPUCHE HUILLICHE WOMEN IN OSORNO PROVINCE

Objective: In order to make available disaggregated information on nutritional state and cardiovascular risk factors among Mapuche Huilliche women, and establish a baseline for future studies and initiatives, a statistically representative sample of Mapuche Huilliche women, heads of households, in rural and urban areas in Osorno Province. Materials and Methods: A non-experimental, cross-sectional, descriptive study design was used. Results: $48 \%$ had a $B M I>30,18.1 \%$ higher than the national average. $42.1 \%$ showed abdominal obesity (waist circumference $>83 \mathrm{~cm}$ ) and $73.5 \%$ showed high risk values of waist-hip and waist-height ratios. The relationship of these indicators to other variables such as age, rural residence, blood pressure and chronic non transmissible diseases is discussed. Conclusions: A high prevalence of cardiovascular risk was found, as well as low perception of nutritional state as related to BMI.

Key words: nutritional state, Mapuche Huilliche women, cardiovascular risk. 


\section{INTRODUCCIÓN}

Aunque las condiciones socioeconómicas y culturales que caracterizan la situación epidemiológica actual, afectan a toda la población chilena ${ }^{1-4}$, pueden impactar de manera muy diferente en los diversos grupos que la componen. La población mapuche huilliche de la Provincia de Osorno, es un grupo especialmente vulnerable debido a una historia marcada por pobreza y segregación socio cultural que ha acompañado a la mayoría de las poblaciones originarias. Según el Censo del año $2002^{5}$ la población huilliche, con 100.660 personas, representa un $14,7 \%$ del total de habitantes de la Región de Los Lagos, concentrándose principalmente en las comunas de San Juan de la Costa, Río Negro y Osorno. Un $43 \%$ de población es no económicamente activa -menor de 15 años y mayor de 65- y un $48 \%$ habita en zonas rurales, donde la actividad principal es la crianza de ganado menor, pequeña agricultura de subsistencia, pesca artesanal y producción de leña y carbón. La emigración del campo a la ciudad está dada principalmente por mujeres jóvenes y solteras en busca de mayores oportunidades laborales. En la ciudad, el $52 \%$ trabaja en forma asalariada, un $33 \%$ en servicio doméstico y un $10 \%$ lo hace por cuenta propia. La tasa de escolaridad de la población huilliche es de nivel básico en el $53,2 \%$, media $28,7 \%$ y superior, $7 \%$; situación desventajosa si se la compara con el promedio nacional donde se encuentra un $40,5 \%, 36 \%$ y $16,6 \%$, respectivamente ${ }^{1}$. La tasa de analfabetismo (8,7\%), representa algo más que el doble del promedio de la población total del país $(4,05 \%)$. Desde el punto de vista antropométrico, la población mapuche huilliche de la Provincia de Osorno se caracteriza por presentar estatura baja y contextura gruesa, especialmente en las generaciones mayores, lo que concuerda con antecedentes antropológicos del siglo recién pasado que dan cuenta de una estatura media de $1,4 \mathrm{~m}$. en los varones y de 1,3 m. en las mujeres ${ }^{6}$. No se encontró información estadística desagregada de las características antropométricas actuales de esta etnia, de su estado nutricional, de la prevalencia de factores de riesgo cardiovascular ni de cómo estos indicadores son percibidos por ellos. Para dar respuesta a estas interrogantes, este estudio se centró en la población adulta fe- menina, dueña de casa, por presentar mejores condiciones de accesibilidad y por considerarla un informante clave, en aspectos alimentarios de ella y de su familia, que no se incluyen en esta publicación. La información obtenida entrega datos que conviene considerar en la atención primaria de salud y en formulación de programas de intervención y promoción de salud que incluyan a la mujer mapuche huilliche.

\section{MATERIAL Y MÉTODO}

Para esta investigación se realizó un levantamiento sincrónico de la información trabajando sobre un universo conformado por las mujeres mapuche huilliche dueñas de casa, que habitan en sectores urbanos y rurales de las siete comunas que conforman la Provincia de Osorno, de acuerdo a la información consignada en el Censo 2002.

Para la selección de la muestra se consideró como población universo a las mujeres que se auto identifican como mapuche huilliche y cuya condición indígena esté reconocida por la Corporación Nacional de Desarrollo Indígena (Conadi) de acuerdo a la legislación vigente? ${ }^{7}$. Otro criterio de selección fue que desempeñaran el rol de dueñas de casa, entendiendo como tal, estar a cargo de la alimentación de su grupo familiar, independiente de si realizan o no trabajo remunerado fuera del hogar. De este modo, la muestra estuvo conformada por 373 unidades muestrales (dueñas de casa mapuche huilliches), tamaño que corresponde a un nivel de confianza de $95 \%$ y un error estimado del $5 \%$ para el universo anteriormente mencionado. Este tamaño se consideró estadísticamente representativo para poblaciones finitas en torno a las 7.000 unidades. El muestreo se realizó por cuotas, con cortes en función al porcentaje de población urbana y rural por cada comuna de la provincia, lo que permitió establecer comparaciones entre las cuotas definidas.

Para determinar el estado nutricional y factores de riesgo de ECNT se utilizaron mediciones antropométricas de peso, talla y perímetros de cintura, cadera y muñeca. Para pesar se utilizó una balanza electrónica portátil Fagor BB-160 para $150 \mathrm{~kg}$. con división de $100 \mathrm{gr}$. La medición de estatura se realizó con tallímetros de pared y la de perímetros se hizo con huincha 
antropométrica flexible e inextensible de $150 \mathrm{~cm}$ con un ciego de $5 \mathrm{~cm}$ para facilitar la maniobra de medición. Con estos datos se determinó índice de masa corporal (IMC), relación cinturacadera $(\mathrm{RCC})$, relación cintura-estatura (RCE) y contextura corporal. Adicionalmente, se midió la presión arterial con esfingomanómetro aneroides con fonendo, adquiridos para este efecto. A quienes presentaron un IMC igual o superior a $30 \mathrm{~kg} / \mathrm{m}^{2}$, definido por la OMS como punto de corte para determinar obesidad, se les ofreció la posibilidad de medir sus niveles de colesterol, triglicéridos y glucosa en sangre capilar. Para ello se utilizó Accu-Chek Go cuyo rango de medición es de 20 - $600 \mathrm{mg} / \mathrm{dl}$. Para la medición de colesterol total y triglicéridos se usó un kit de Accutrend GCT cuyos rangos de medición son $150-300 \mathrm{mg} / \mathrm{dl}$ y $70-600 \mathrm{mg} / \mathrm{dl}$, respectivamente. Se incluye la información de este subgrupo. Los puntos de corte utilizados para cada indicador fueron los siguientes:

\begin{tabular}{|c|c|c|}
\hline IMC (Kg/m2) & \multicolumn{2}{|c|}{$\begin{array}{ll}\text { Bajo: } & <18,5 \\
\text { Normal: } & 18,5-24,9 \\
\text { Sobrepeso: } & 25-29,9 \\
\text { Obesidad: } & 30-39,9 \\
\text { Obesidad mórbida: } \geq 40 \text { o más. }\end{array}$} \\
\hline $\begin{array}{l}\text { Circunferencia de cintura (CC) (obesidad abdominal } \\
3 \text {, criterio determinado en base a ENS 2003) }\end{array}$ & \multicolumn{2}{|l|}{$\geq 83 \mathrm{~cm}$} \\
\hline $\begin{array}{l}\text { Relación cintura cadera, RCC (circunferencia cintura } \\
\text { en } \mathrm{cm} / \text { circunferencia cadera en } \mathrm{cm} \text { ) }\end{array}$ & $\begin{array}{l}\text { Alto: } \\
\text { Mediano: } \\
\text { Bajo: }\end{array}$ & $\begin{array}{l}\geq 0,85 \\
0,80 y<0,85 \\
<0,80\end{array}$ \\
\hline $\begin{array}{l}\text { Relación cintura estatura, RCE (circunferencia cintura } \\
\text { en } \mathrm{cm} / \text { estatura en } \mathrm{cm} \text { ) }\end{array}$ & \multicolumn{2}{|c|}{$\begin{array}{ll}\text { Normal: } & <0,55 \\
\text { Riesgo cardiovascular: } & \geq 0,55\end{array}$} \\
\hline $\begin{array}{l}\text { Contextura corporal ( } \mathrm{cm} \text { talla/ } \mathrm{cm} \text { circunferencia de } \\
\text { muñeca) }\end{array}$ & $\begin{array}{l}\text { Pequeña: } \\
\text { Media } \\
\text { Gruesa }\end{array}$ & $\begin{array}{l}>11 \\
10,1 \text { a } 11 \\
<10,1\end{array}$ \\
\hline $\begin{array}{l}\text { Glicemia capilar (HG) para mediciones en cualquier } \\
\text { momento del día }\end{array}$ & Normal: & $\leq 140 \mathrm{mg} / \mathrm{dl}$ \\
\hline Colesterol total en sangre capilar & Normal: & $<200 \mathrm{mg}$ \\
\hline Triglicéridos en sangre capilar & Normal: & $<150 \mathrm{mg}$ \\
\hline Presión arterial & Normal: & $<140 /<90 \mathrm{mmHg}$ \\
\hline Actividad física multidimensional & \multicolumn{2}{|c|}{$\begin{array}{l}\text { Sedentarismo: }<150 \text { min. de activi- } \\
\text { dad moderada/semana o } \leq 20 \text { min. } \\
\text { actividad intensa al menos } 3 \text { veces a } \\
\text { la semana }\end{array}$} \\
\hline
\end{tabular}

De la encuesta de calidad de vida 2006 se seleccionaron aquellas preguntas relacionadas con actividad física de tipo laboral y recreativo o intencional y las imágenes gráficas para conocer la percepción de su estado nutricional. Adicionalmente, se incluyeron preguntas diseñadas especialmente con items semi estructurados en función del piloto de validación realizado en la población objetivo del proyecto. Para el análisis de concordancia entre diagnóstico nutricional y la autopercepción del peso, se utilizó el coeficiente kappa ponderado. El procesamiento y análisis de datos se realizó utilizando los softwares SPSS versión 15.0 y planilla de cálculos Microsoft Office Excel, versión 2007. Para apoyar la valoración de los datos se utilizan las re- 
ferencias nacionales de la Encuesta de Salud 2009-2010 (ENS 2009-2010) ${ }^{4}$.

\section{Consideraciones éticas}

Cada participante recibió información inicial, verbal y escrita respecto a los objetivos y procedimientos del proyecto y formalizó su voluntad de participar firmando un consentimiento. Según lo comprometido, el conjunto de resultados fue entregado a Conadi, organismo patrocinante y tanto el proyecto como los instrumentos a utilizar fueron revisados y aprobados por el comité de ética de la Universidad de Los Lagos.

\section{RESULTADOS}

\section{Características generales de la muestra}

La muestra, estadísticamente representativa de la población femenina mapuche huilliche (MMH) mayor de 15 años de la Provincia de Osorno, estuvo conformada por 373 jefas de familia, con- centrándose mayoritariamente en las categorías de 20 a 64 años (92,8\%). Un 55,8\% habita en zonas urbanas y un $44,2 \%$ en zonas rurales. La actividad laboral no remunerada caracterizó a la mayoría de las encuestadas (72,9\%) La escolaridad es de nivel básico para el $48,5 \%$, de nivel medio para el $36,5 \%$ y de nivel superior para el $10,5 \%$ restante. El analfabetismo afecta a un $4,56 \%$ de la muestra, muy similar a la tasa de $4,25 \%$ calculado para la mujer en Chile. De 17 mujeres calificadas como analfabetas, solo una tiene menos de 40 años; 8 tienen entre 40 y 59 años y 8 más de 60 años, lo que estaría reflejando un efecto positivo del aumento progresivo de la cobertura escolar y de los programas de nivelación de estudios de las últimas décadas.

\section{Características antropométricas}

La MM-H tiene -en promedio- $4,8 \mathrm{~cm}$ de talla menos que la mujer en $\mathrm{Chile}^{4}$. Su peso promedio es mayor en $2,7 \mathrm{~kg}$ y, por lo tanto, la circun-

Tabla 1. Resumen de resultados antropométricos e indicadores de riesgo cardiovascular en la MM-H y la población femenina a nivel país según ENS 2009-2010.

\begin{tabular}{|c|c|c|}
\hline $\begin{array}{l}\text { Características antropométricas, } \\
\text { indicadores de riesgo y presión arterial }\end{array}$ & $\begin{array}{l}\text { Muestra MM-H } \\
(\mathrm{N}=373)\end{array}$ & $\begin{array}{c}\text { Mujer país } \\
\text { (ENS 2009-2010) }\end{array}$ \\
\hline Peso promedio (kg) & $70,2(51,8-88,6)$ & $67,5(66,6-68,4)$ \\
\hline Talla promedio $(\mathrm{cm})$ & $151,3(145,7-156,8)$ & $156,1(155,5-156,7)$ \\
\hline Circunferencia cintura promedio $(\mathrm{cm})$ & $92,6(79,6-105,6)$ & $88,2(87,3-89,1)$ \\
\hline Complexión corporal “Grande” (\%) & 63,4 & $\mathrm{~s} / \mathrm{i}$ \\
\hline IMC $\left(\mathrm{kg} / \mathrm{m}^{2}\right)$ promedio & $30,8(21,9-39,7)$ & $27,9(27,3-28,4)$ \\
\hline $\begin{array}{l}\text { Razón cintura estatura RCE (cm cintura/ } \\
\mathrm{cm} \text { estatura) promedio }\end{array}$ & $0,61(0,5-0,7)$ & $\mathrm{s} / \mathrm{i}$ \\
\hline $\begin{array}{l}\text { Razón cintura cadera RCC (cm cintura/cm } \\
\text { cadera) promedio }\end{array}$ & $0,92(0,8-1,00)$ & $\mathrm{s} / \mathrm{i}$ \\
\hline Presión arterial promedio $(\mathrm{mmHg})$ & $120 / 80 \mathrm{mmHg}$ & $121,9 / 73,9 \mathrm{mmHg}$ \\
\hline Prevalencia & $\begin{array}{l}\text { Muestra MM-H } \\
\quad(\mathrm{N}=373)\end{array}$ & $\begin{array}{c}\text { Mujer país } \\
\text { (ENS 2009-2010) } \\
\end{array}$ \\
\hline Circunferencia cintura $\geq 83 \mathrm{~cm}$ & $78,4 \%$ & $60,5 \%$ \\
\hline RCE (Alto riesgo) & $73,5 \%$ & $\mathrm{~s} / \mathrm{i}$ \\
\hline RCC (Alto riesgo) & $73,5 \%$ & $\mathrm{~s} / \mathrm{i}$ \\
\hline Obesidad (IMC $\geq 30<40$ ) & $40,2 \%$ & 30,7 \\
\hline Obesidad mórbida (IMC $\geq 40$ ) & $8,6 \%$ & 3,3 \\
\hline Presión arterial $\geq 140 / \geq 90 \mathrm{mmHg}$ & $24,7 \%$ & $25,3 \%$ \\
\hline Sedentarismo multidimensional & $\begin{array}{c}20,6 \% \text { (rural) } \\
24,0 \% \text { (urbano) }\end{array}$ & $31,7 \%$ \\
\hline
\end{tabular}

s/i: sin información 
Tabla $\mathbf{N}^{\circ}$ 2. Estado nutricional de la MM-H de acuerdo a IMC, según grupos de edad.

\begin{tabular}{lcccccc}
\hline Edad & $\mathbf{N}$ & $\begin{array}{c}\text { Bajo } \\
\%\end{array}$ & $\begin{array}{c}\text { Normal } \\
\%\end{array}$ & $\begin{array}{c}\text { Sobrepeso } \\
\%\end{array}$ & $\begin{array}{c}\text { Obesidad } \\
\%\end{array}$ & $\begin{array}{c}\text { Ob. Mórbida } \\
\%\end{array}$ \\
\hline $15-44$ & 235 & 0,9 & 22,6 & 37,9 & 32,8 & 6,0 \\
$45-64$ & 119 & 0,8 & 7,6 & 25,2 & 54,6 & 11,8 \\
$\geq 65$ & 19 & 0,0 & 10,5 & 26,3 & 42,1 & 21,1 \\
Total & 373 & 0,8 & 17,2 & 33,2 & 40,2 & 8,6 \\
\hline
\end{tabular}

Morbilidad auto reportada y tratamiento.

ferencia de cintura y los indicadores complejos como IMC y RCE están más distantes del rango normal. El IMC promedio es de $30,8 \mathrm{~kg} / \mathrm{m}^{2}$, correspondiente a la categoría de "obesidad" y es superior al IMC promedio de la mujer en Chile $\left(27,9 \mathrm{~kg} / \mathrm{m}^{2}\right)$, que se ubica en el rango de "sobrepeso". El promedio de circunferencia de cintura es de $92,6 \mathrm{~cm}$, encontrándose que un $78,4 \%$ supera los $82 \mathrm{~cm}$ establecidos según la Encuesta de Salud 2003 para determinar obesidad abdominal en la mujer chilena². Esto supera en 18 puntos porcentuales el promedio de la mujer en Chile (Tabla 1). En concordancia con lo anterior, la relación cintura/estatura es de 0,61, superando también la razón de 0,55 definida como límite para este indicador. Otros factores como presión arterial elevada y sedentarismo multifuncional, mostraron valores más bajos que el promedio femenino nacional.

\section{Indice de masa corporal según ruralidad y grupos de edad}

Se encontró un alto índice de obesidad que equivale a 1,3 y 2,6 veces, respectivamente, el promedio de obesidad y obesidad mórbida que se describe en la ENS 2009-2010 (Tabla 1). Al analizar según ruralidad, se encontró que en la zona urbana hay un mayor porcentaje de sobrepeso ( $36,1 \%$ vs. $29,7 \%$ en zona rural) y en la zona rural es mayor la obesidad general $(57,6$ vs. $41,9 \%$ en zona urbana).

A su vez, la magnitud del exceso de peso corporal va aumentando con la edad de modo que la mayor proporción de mujeres con sobrepeso se encuentra en el grupo de 15 a 44 años. La mayoría de las mujeres que presenta obesidad, se encuentra en el grupo de 45-64 años y la mayor proporción de mujeres con "obesidad mórbida" se encuentra en el grupo de mayores de 65 años (Tabla 2).

Del total de la muestra, 72 mujeres $(19,5 \%)$ reportaron diagnóstico de hipertensión (HTA) y de ellas, un $93,1 \%$ se encuentra en tratamiento en el sistema de atención primaria de salud. De las 182 mujeres con IMC igual o mayor a 30, sólo 14 afirmaron tener diagnóstico de obesidad y de ellas, 12 están bajo control en servicios de atención primaria. El diagnóstico de diabetes fue reportado por 40 encuestadas $(10,5 \%$ del total de la muestra) de las cuales, $32(82,5 \%)$ afirman que están siendo tratadas regularmente en el consultorio. Otras enfermedades referidas por las encuestadas, en orden descendente, fueron las de tipo reumático como artritis y artrosis y en segundo orden, depresión y afecciones al hígado y las vías biliares. El uso de recursos propios de la cultura mapuche-huilliche en el cuidado de su salud, es muy bajo según las respuestas obtenidas. Solo una mujer con diagnóstico de diabetes y otra con diagnóstico de HTA señalaron la medicina mapuche como única opción de tratamiento.

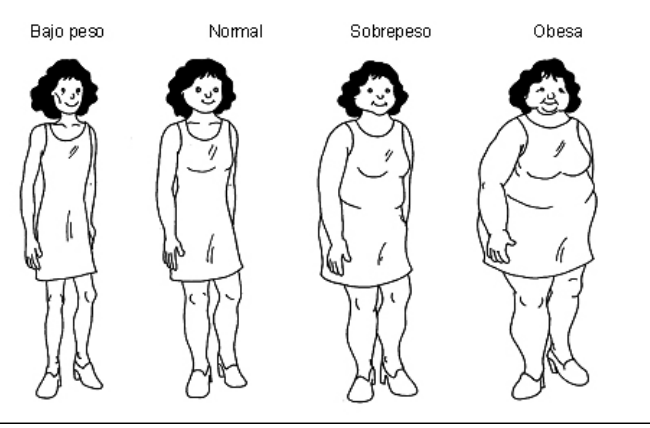

Anexo 1. Figuras utilizadas para describir la autopercepción corporal de las encuestadas. Fuente: Encuesta Nacional de Salud 2003. Chile. 
Tabla 3. Número y porcentaje de enfermedades diagnosticadas en los niveles de Alto Riesgo de cada indicador.

\begin{tabular}{lccc}
\hline \multirow{2}{*}{$\begin{array}{c}\text { Indicador de riesgo } \\
\text { cardiovascular }\end{array}$} & \multicolumn{2}{c}{ Reporte de enfermedades diagnosticadas } \\
\cline { 2 - 4 } & $\begin{array}{c}\text { HTA } \\
(\mathbf{n = 7 1 )} \\
\%\end{array}$ & $\begin{array}{c}\text { Diabetes } \\
(\mathbf{n = 3 8 )}\end{array}$ & $\begin{array}{c}\text { Obesidad } \\
(\mathbf{n = 1 6 )} \\
\mathbf{N}^{\circ} \text { CASOS }\end{array}$ \\
\hline IMC $\geq 30$ (Obesidad I, II, III) & 59,7 & 72,5 & 14 \\
CC $\geq 82$ (Obesidad Abdominal) & 88,7 & 94,7 & 15 \\
RCC $\geq 0,85$ (Riesgo Alto) & 80,6 & 84,6 & 13 \\
RCE $\geq 0,55$ (Riesgo Alto) & 90,1 & 89,5 & 16 \\
\hline
\end{tabular}

** No se expresa como porcentaje por tratarse de un "n" reducido.

El resumen presentado en la tabla anterior permite destacar que el indicador RCE concentra en el nivel de alto riesgo a la mayoría de las enfermedades reportadas por las encuestadas. De los cuatro indicadores presentados, el IMC $\geq 30$ mostró una menor sensibilidad por cuanto concentró el menor número de enfermedades crónicas diagnosticadas.

\section{Percepción del peso corporal}

Mediante el uso de 4 figuras (Anexo 1) que representan distintos grados de relación de peso para la talla (bajo peso, normal, sobrepeso y obesidad), se encontró que solo un 13,3\% de las mujeres obesas se consideró como tal. La mejor concordancia se observó en las personas calificadas con peso "normal" donde la coincidencia alcanzó a $71,9 \%$, bajando a $36,8 \%$ en las personas con sobrepeso. La situación más frecuente estuvo dada por subestimación del peso de tal modo que cerca de la mitad de las personas con sobrepeso considera que su peso es "normal" y un $63,5 \%$ de las mujeres obesas considera que solo presenta sobrepeso (índice Kappa 0,074 o insignificante).

\section{Control del exceso de peso}

Un $99 \%$ de las encuestadas considera dañino el exceso de peso corporal, pero el 56\% no hace nada para controlarlo. El 44\% restante, que realiza alguna acción para controlar su peso, opta por "regular la ingesta comiendo menos o haciendo dieta" $(27,6 \%)$, "hacer ejercicio (intencionadamente)" $(7,2 \%)$ o apoyarse con automedicación, homeopatía o agua de hierbas $(2,9 \%)$. Solo un 4,8\% busca tratamiento médico o de otro profesional. En la categoría "otros" las respuestas más frecuentes fueron en orden descendente "comer sin sal", "tomar harta agua", "comer más verduras" y "no cenar". Estos resultados resultan similares a lo reportado en la ECV 2006 donde se encontró que un $57,9 \%$ de las mujeres no hace nada y un 42,1 realiza alguna acción para controlar su peso.

\section{Exámenes bioquímicos}

De acuerdo al diseño del proyecto, se realizaron mediciones en sangre sólo en el grupo de mujeres con $I M C \geq 30$. De 170 datos válidos en la medición de TG, más de la mitad $(56,5 \%)$ presentó niveles $\geq 150 \mathrm{mg}$ y un $40,4 \%$ tuvo niveles elevados de CT (n válido=156). El hemoglucotest, con 168 datos válidos, resultó elevado en 20 personas obesas, de las cuales solo 9 tenían diagnóstico de diabetes y se encontraban en tratamiento.

\section{DISCUSIÓN}

La evaluación nutricional de las MM-H, basada en IMC mostró una alta prevalencia de obesidad y obesidad mórbida, superando de manera importante el promedio para la mujer chilena. Por su parte, los valores que exceden los límites recomendables de circunferencia de cintura, relación cintura-cadera y relación cintura-estatura mostraron una frecuencia mayor que el promedio nacional para cada indicador y concentraron en la categoría de alto riesgo a la mayoría de 
las enfermedades crónicas no transmisibles que les han sido diagnosticadas a las integrantes del grupo participante.

Al analizar por separado cada indicador de riesgo se encontró que la RCE mostró la mejor relación entre valores de alto riesgo y cantidad de enfermedades reportadas, lo que concuerda con la conclusión de otros autores que han estudiado su capacidad predictiva de riesgo cardiovascular para la población chilena ${ }^{8}$. En el grupo estudiado, la baja estatura es una característica relevante que puede ser consecuencia más de su historia de pobreza y vulnerabilidad, por lo que adquiere relevancia el uso de un indicador que considera esta variable en su definición. A ello cabe agregar la conveniencia práctica de disponer de un indicador de riesgo que no requiere la determinación del peso corporal para aquellas situaciones en que no se dispone de balanzas debidamente calibradas 0 donde no se cuente con las mínimas condiciones de infraestructura y confort requeridas para ello. El IMC $\geq 30$ resultó ser el indicador menos efectivo para detectar al grupo que reportó diagnóstico de enfermedades crónicas.

En cuanto a la autopercepción del estado nutricional, existe una baja concordancia con la calificación de categorías de IMC correspondientes a exceso en cualquier grado y según el auto reporte de enfermedades diagnosticadas, destaca la baja tasa de diagnóstico de obesidad. La falta de conciencia de su propio estado nutricional podría influir en retrasar el inicio de acciones de reparación como moderar la ingesta o buscar apoyo profesional. Por otra parte, su rol como dueñas de casa y habituales responsables de la alimentación familiar, hacen que su percepción frente al exceso de peso y la valoración que hagan de la alimentación prudente y saludable tenga un efecto multiplicador.

Los resultados encontrados reflejan que la $\mathrm{MM}-\mathrm{H}$ tiene indicadores de riesgo cardiovascular más altos que el promedio de la población femenina de todo el país y la mayoría desconoce esta condición. Es necesario aumentar los esfuerzos por detectar los casos de alto riesgo, ofrecer opciones de tratamiento y seguir realizando estudios que permitan contar con información desagregada -y referida a ambos sexospara mejorar la orientación de programas de atención y prevención que incluyan a este grupo de la población.

\section{REFERENCIAS}

1. Chile. Ministerio de Salud. II Encuesta Nacional de Calidad de Vida y Salud. Santiago: MINSAL; 2006.

2. Chile. Ministerio de Salud, Instituto Nacional de Estadísticas (Chile). Encuesta Nacional de Salud (ENS) 2003. Santiago: MINSAL; 2004.

3. Vio F, Albala C, Kain J. Nutrition transition in Chile revisited: mid-term evaluation of obesity goals for the period 2000-2010. Public Health Nutr. 2008 Apr;11(4):405-12.

4. Chile. Ministerio de Salud. Encuesta Nacional de Salud 2009-2010. Santiago: Minsal.

5. Instituto Nacional de Estadísticas (Chile). Programa Orígenes (Mideplan/BID). Estadísticas sociales de los pueblos indígenas en Chile: Censo 2002. Santiago: INE; 2005.

6. Alcamán E. Los Mapuche Huilliche del Futahullimapu Septentrional. Revista de Historia Indígena, №2, Dic 1997. Páginas 21-75. Universidad de Chile.

7. Ley indígena, ley no. 19.253(actualizada): Convenio no. 169 sobre"pueblos indígenas y tribales en países independientes" de la Organización Internacional del Trabajo, OIT. Corporación Nacional de Desarrollo Indígena, Conadi, 2009.

8. Koch E, Díaz C, Romero T, Kirschbaum A, Martínez L, Paredes M, Taylor A. Razón cintura-estatura como un predictor de mortalidad en población chilena: un estudio de 8 años de seguimiento en la cohorte del proyecto San Francisco. Rev Chil Cardiol. 2007;26(4):23-35

9. World Health Organization. Obesity: preventing and managing the global epidemic. Report of a WHO Consultation (WHO Technical Report Series 894). Geneva: WHO. 2000.

10. Atalah E, Urteaga C, Rebolledo A. Autopercepción del estado nutricional en adultos de Santiago. Rev Méd Chile [en línea]. 2004 [consultado el 9 de febrero de 2010];132(11):1383-1388. Disponible en: http:/l www.scielo.cl/pdf/rmc/v132n11/art07.pdf

11. Franquelo P, Serrano S, Moya P, Buendía J, Sánchez M, Solera M et al. Asociación entre distintas medidas de Composición Corporal y Factores de Riesgo Cardiovascular en población adulta. Rev Clin Med Fam [en línea]. 2008 Jun [consultado el 28 de mayo de 2012];2(4):149-155. Disponible en: http://scielo.isciii.es/scielo.php?script=sci_arttext\&pid=S1699$695 \times 2008000200003 \& \operatorname{lng}=e s$

12. Mujica V, Leiva Elba, Rojas E, Díaz N, Icaza G, Palomo I. Discordancia en autopercepción de peso en población adulta de Talca. Rev Méd Chile [en línea]. 2009 Ene [consultado el 28 de mayo de 2012];137(1):76-82. Disponible en: http://www. scielo.cl/scielo.php?script=sci_arttext\&pid=S003498872009000100011\&lng=es. 
13. Thompson J, Tantleff-Dunn S. Assessment of body image disturbance in obesity. Obes Res. 1998;6:375-7.

14. Paeratakul S, White M, Williamson D, Ryan D, Bray G. Sex, race/ethnicity, socioeconomic status, and BMI in relation to self-perception of overweight. Obes Res. 2002:10:345-50.

15. Donath SM. Who's overweight? Comparison of the medical definition and community views. Med J Aust. 2000;172:375-7.
16. Foerster R. Los huilliches y el mar. Proyecto Fondecyt-No 1950352. 1995.

17. Alcafuz A. Kumiyal, comida mapuche huilliche. Programa Nacional de Salud para Pueblos Indígenas, Osorno; 2004.

Recibido: 25 de diciembre de 2011 Aprobado : 11 de mayo de 2012 